\title{
Vers une théorie et une critique de l'architecture
}

For a Theory and an Architectural Criticism

\section{Manuel J. Martín-Hernández}

Traducteur : Lilian Périer

\section{(Q) OpenEdition}

\section{Journals}

Édition électronique

URL : http://journals.openedition.org/crau/306

DOI : $10.4000 /$ crau.306

ISSN : 2547-5746

\section{Éditeur}

Éditions du patrimoine

\section{Édition imprimée}

Date de publication : 1 décembre 2009

Pagination : $99-112$

ISBN : 978-2-85822-944-4

ISSN : 1296-4077

\section{Référence électronique}

Manuel J. Martín-Hernández, « Vers une théorie et une critique de l'architecture », Les Cahiers de la recherche architecturale et urbaine [En ligne], 24/25 | 2009, mis en ligne le 01 septembre 2017, consulté le 30 avril 2019. URL : http://journals.openedition.org/crau/306 ; DOI : 10.4000/crau.306 
La théorie a signifié une série de règles pour l'interprétation et l'action aspirant à expliquer et à comprendre la réalité.

Or dans le domaine de l'architecture, elle n'est plus aujourd'hui une tendance prioritaire, probablement parce qu'elle est aussi un moyen de questionner et de critiquer cette architecture. Pourtant, la réalité est construite par la théorie qui a besoin d'une certaine distance et d'une certaine durée que la pratique active $n$ 'autorise guère. Dans cette période "post-critique ", pratique et critique sont séparées et, comme le montre ici M. J. Martín-Hernández, la criticalité a perdu sa place au motif de son inutilité apparente. Mais n'est-il pas nécessaire de justifier et de transmettre les choix architecturaux, de partager un langage commun? N'est-il pas nécessaire de rechercher une cohérence, de construire un savoir sur l'architecture?

\title{
Vers une théorie et une critique de l'architecture'
}

\author{
MANUEL J. MARTÍN-HER NÁNDEZ
}

\section{Contre la théorie}

Dans les années 1980, les "néo-pragmatiques » Steven Knapp et Walter Benn Michaels ont écrit un article provocateur dans le champ des études littéraires - «Against Theory ${ }^{2} »-$, qui a eu un important retentissement aux États-Unis. Dans son acception classique, la théorie forge une série de règles pour l'interprétation et l'action qui visent à expliquer et à faire comprendre des réalités multiples avec relativement peu d'instruments, qui se trouvent être le plus souvent les idées des classes et des pouvoirs dominants. «La théorie se situe toujours au début ou à la fin de la pensée, fournissant les principes premiers à partir desquels les hypothèses, les lois et les méthodes peuvent être déduites, ou résumant, codifiant et schématisant la pratique comme simple moyen de justification ${ }^{3}$. » Or, le propos de Knapp et Michaels consistait à nier l'idée même de théorie. Si être un théoricien signifie croire qu'il est possible de choisir parmi des méthodes alternatives et que certaines " croyances » sous-tendent une pratique particulière (théorie de la réception, structuralisme, herméneutique, déconstruction, psychanalyse, marxisme, féminisme...), pour les deux auteurs, la théorie ne serait rien de plus qu'une tentative pour échapper à la

1. Une première version en anglais de cet article a été publiée sous le titre «For (a) theory (of architecture) » dans The Journal of Architecture, vol. XIII, $n^{\circ} 1$, février 2008, pp. 1-7. Le texte proposé dans ce dossier des Cahiers correspond à une version révisée et augmentée.

2. À l'origine dans le $n^{\circ} 4$, vol. VIII de l'été 1982 de Critical Inquiry, réédité comme texte de base de la discussion dans W. J. T. Mitchell, Against Theory. Literary Studies and the New Pragmatism, Chicago/Londres, The University of Chicago Press, 1985, pp. 11-30.

3. W. J. T. Mitchell, «Introduction», ibid., p. 7. 
pratique parce que, de leur point de vue, il n'est pas possible de se situer hors de la pratique.

Vingt ans plus tard, coïncidant avec le fort mouvement anti-guerre international du printemps 2003, Critical Inquiry - la revue qui est déjà à l'origine du débat - explorait à nouveau la question du futur de la théorie et de la critique. Ainsi le directeur de la rédaction, W. J. T. Mitchell, s'interrogeait-il: "Que peut faire le pouvoir relativement faible de la théorie critique, face à une telle crise? ", et devrait-on considérer que « la grande ère de la théorie est désormais derrière nous et que nous sommes entrés aujourd'hui dans une période de pusillanimité, de repli et (au mieux) de construction empirique ${ }^{4}$ ? » Les réponses apportées dans le débat soulignaient l'ambivalence de la théorie ainsi que la difficulté en cette période d'adopter une position « radicale ».

W. J. T. Mitchell, Against Theory, 1985.

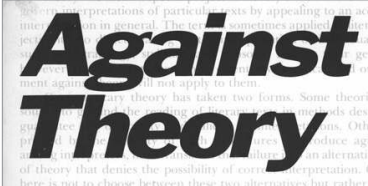

Literary Studies and the New Pragmatism

Edited by W.J.T. Mitchell
En avril 1999, dans le champ de l'architecture, Francesco Dal Co avait de son côté amorcé un débat autour de la vacuité de la théorie dans la revue Casabella, qu'il dirigeait alors. Selon Dal Co, la théorie n'étant ni le projet, ni l'histoire ni la critique, celle-ci devrait en réalité occuper la place où se croisent ces trois disciplines: c'est-à-dire là où les architectes pourraient être questionnés sur le sens de leur propre activité, et interrogés sur « la signification et les modalités de la pratique architecturale, afin d'éliminer les problèmes qui ne sont pas inhérents à l'expérience subjective, mais relèvent de l'objectivité d'un mode de fonctionnement commun ${ }^{5}$ ", et en essayant de préciser ce qui permet, aux uns et aux autres, de parler d'architecture et de se comprendre. Mais pour Dal Co, bien peu d'architectes se seraient véritablement engagés dans cette tâche.

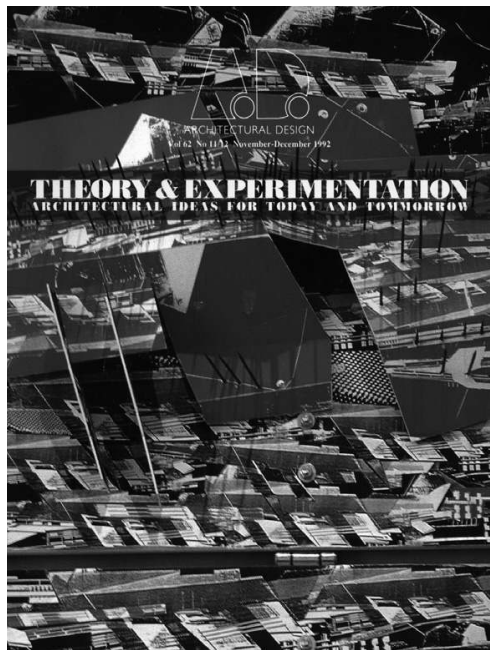

Architectural Design n'11/12, 1992.
4. W. J. T. Mitchell, « Medium Theory: Preface to the 2003 Critical Inquiry Symposium », Critical Inquiry, vol. XXX, $n^{\circ} 2$, hiver 2004, p. 326. Dans ce volume sont regroupées les communications de la conférence "The Future of Criticism », qui a eu lieu à Chicago les 11 et 12 avril 2003. Voir sur le site: http://criticalinquiry.uchicago.edu/. Voir aussi I'article du New York Times du 19 avril 2003, " The Latest Theory Is That Theory Doesn't Matter ", et I'article du Boston Globe du 20 avril 2003,

"Crisis Theory".

5. Francesco Dal Co, «Teoria, parola cava? ", Casabella n 666, 1999, p. 32. 
Il ne fait aucun doute qu'au cours des décennies passées les architectes ont eu besoin de la théorie pour mettre en valeur leur œuvre. De fait, les architectes-théoriciens ont été les plus intéressants: Aldo Rossi, Robert Venturi, Peter Eisenman, Vittorio Gregotti, Rafael Moneo, John Hejduk ou encore Rem Koolhaas... Mais aujourd'hui, le discrédit de la théorie architecturale est tel qu'il nous conduit à écouter, sans étonnement, certaines

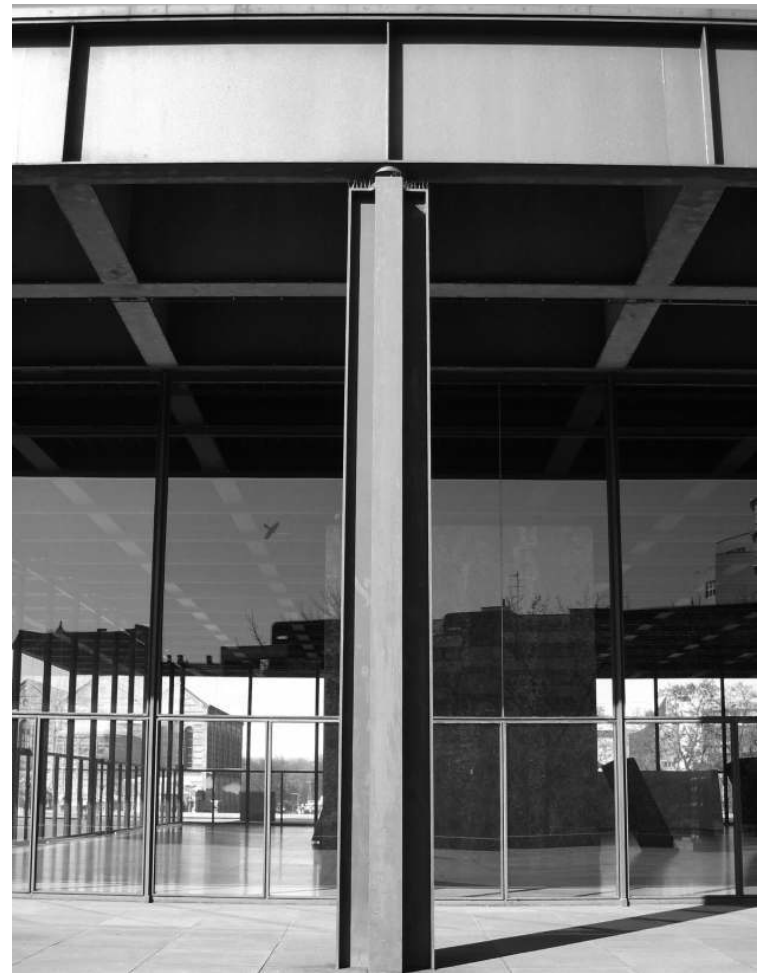

Ludwig Mies van der Rohe, Neue Nationalgalerie, Berlin, 1962-1968.

Photo DR. déclarations de la part d'architectes du star system comme Jacques Herzog (d'Herzog et de Meuron, lauréats du prix Pritzker, désormais incontournables et sollicités dans le monde entier) qui, à une question posée par Jeffrey Kipnis sur son rapport à la théorie, affirme ceci : "Ce qui perdure, ce qui influence l'architecture, ce qui transforme l'architecture en architecture, c'est l'œuvre: les bâtiments et les projets. Nous ne nous

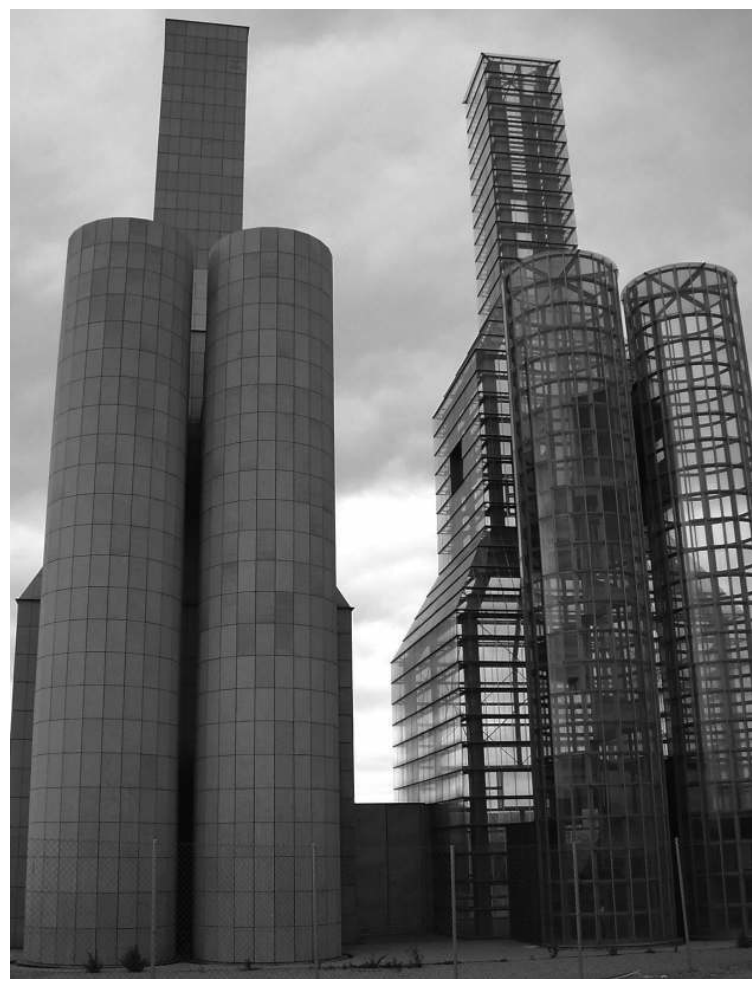

John Hedjuk et Antonio

Sanmartín, Tribute Towers,

Cité de la culture galicienne,

Saint-Jacques-de-Compostelle, 1999-2003. Photo DR. 
rappelons aucun texte qui ait changé notre mode de penser, qui ait signifié quelque chose dans notre architecture. Les mots et les textes sont de simples séductions. Merveilleux, mais manquant de sens: ils n'offrent, d'aucune manière que ce soit, la moindre aide. II n'y a aucune exception à cela ${ }^{6}$. » II ne pouvait pas en être autrement. Leur architecture est le fruit d'une idée (différente pour chaque projet et résultat direct d'un remueméninges au sein de l'agence) sans références (apparemment, il n'y a pas de sources pas plus qu'une inspiration ouvertement reconnue), une " création pure " par conséquent. C'est de la peau et de la cosmétique ${ }^{7}$. Par ailleurs, leur mise en œuvre requiert une technique soigneusement élaborée, qui s'appuie sur des processus d'expérimentation, à partir de maquettes à grande échelle. Jacques Herzog et Pierre de Meuron ne sont pas les seuls, il s'agit d'un exemple parmi d'autres. Les réalisations apparemment les plus exaltantes de l'architecture occidentale font appel aux nouvelles technologies et aux nouveaux matériaux, aux systèmes et modèles produits par ordinateur; et, grâce à l'économie postcapitaliste (y compris en plein milieu - ou en dépit d'une crise économique majeure), elles se produisent dans un contexte de pratiques constructives et spéculatives de plus en plus obsessionnelles, sans freins ni scrupules $^{8}$. Face à cette situation, la critique est en retrait: elle ne dispose d'aucune ouverture possible et c'est la raison pour laquelle, du moins en architecture, il n'y a pas de place non plus pour la théorie.

Dans "Le point de vue scolastique », une communication présentée à l'Université libre de Berlin en 1989, Pierre Bourdieu parlait de l'homo scholasticus ou acade- micus qui, bénéficiant des moyens nécessaires (temps libre - c'est-à-dire skholè-, compétences acquises par l'étude et disposition à investir ce temps en possibles loisirs), produit des discours libérés des limites coercitives, conjoncturelles ou des simples urgences auxquelles la pratique doit répondre 9 . Le propos n'est pas d'opposer la théorie à la réalité, bien au contraire, car la réalité est construite par la théorie, ou, dit autrement: c'est la théorie qui révèle ou constitue une réalité chaque fois que celle-ci entre en action. Mais une certaine distance est nécessaire pour la comprendre, que la pratique directe ne permet pas. La réflexion théorique n'est donc pas possible lorsqu'on est impliqué en permanence dans la résolution de problèmes immédiats. En cela, le « cynisme » d'un Herzog, auquel il a été fait allusion, a toute sa raison d'être. Ce qui est en question, ce n'est pas le fait que la théorie lui soit utile ou pas, mais le fait que la contemplation sereine dans laquelle se situe la theoría ne fasse pas partie de ses priorités. Un exemple parmi d'autres. Car force est de constater que l'attitude fréquente chez les architectes consistant à nier ou à éviter toute référence à leurs sources - au nom du mythe de la «création » architecturale - ne favorise pas l'établissement d'une théorie, quelle qu'elle soit.

Or, comme le rappelle Alberto Pérez-Gómez, l'architecture n'est pas seulement une question esthétique ou technologique mais fondamentalement éthique, étant donné la dimension politique qu'implique sa conceptualisation en tant que bien commun, quand on la définit comme productrice d'espaces destinés à la stabilité et à l'équilibre des hommes ${ }^{10}$. Par conséquent, la théorie est nécessaire: une instance critique permettant $d$ 'inter-
6. Jeffrey Kipnis, « Una conversación con Jacques Herzog $(\mathrm{H} \&$ deM)/A conversation with Jacques Herzog (H \& deM) »,

El Croquis no 84, 1997, p. 18.

7. J. Kipnis, «La Astucia de la Cosmética/The Cunning of Cosmetics », ibid., pp. 22-28.
8. Voir la critique virulente du travail de certains architectes contemporains et le rapport entre l'architecture, l'argent et la politique, dans l'ouvrage de Deyan Sudjic, The Edifice Complex: How the Rich and the Powerful Shape the World, New York, Penguin Press, 2005.

9. Pierre Bourdieu, «Le point de vue scolastique ", Raisons pratiques. Sur la théorie de l'action, Paris, Seuil, 1994 pp. 219-236.
10. Alberto Pérez-Gómez, «The Case for Hermeneutics as Architectural Discourse », in Halina Dunin-Woyseth et Kaj Noschis (dir.), Architecture and Teaching. Epistemological Foundations, EAAE, Lausanne,

Comportements, 1998, p. 23. 
préter la réalité et d'imaginer un possible futur à atteindre, après une négociation qui est toujours une expression de cette tâche politique.

\section{Pourquoi la critique?}

Ce que l'on appelle " architecture critique » vient directement de la notion de "théorie critique », formulée à partir des interprétations post-marxistes de l'École de Francfort: ces interprétations étaient opposées à la raison instrumentale dominante, par une pensée critique qui défendait l'introduction de l'expérience vitale et de la vie quotidienne, au point de se constituer en autoconscience de l'époque et en dénonciation des rapports de pouvoir ${ }^{11}$. La traduction architecturale de cette " théorie critique " a été explicitée notamment par Manfredo Tafuri et par ses collaborateurs de l'École de Venise. Leur préoccupation était de défendre l'architecture en tant qu'instrument de culture, élément complémentaire des rapports de production, face à la vision exclusive de l'architecture comme forme autonome, limitée à la réalisation technique (et artistique) d'une nécessité fonctionnelle. Assurément, I'architecture produit un certain type de connaissance sur la culture et sur l'architecture elle-même ${ }^{12}$, mais la proposition tafurienne allait plus loin: cette « critique » assumait la forme d'une résistance dialectique, d'une « dénonciation, d'un démasquage, d'un jugement négatif » de la manipulation fallacieuse des discours issus de la construction capitaliste du territoire ${ }^{13}$. En dernier recours, I'unique issue était le refuge dans la théorie et dans l'histoire et, par conséquent, le renoncement à la praxis, à l'architecture elle-même (remplacée ici par une "pratique théorique»).

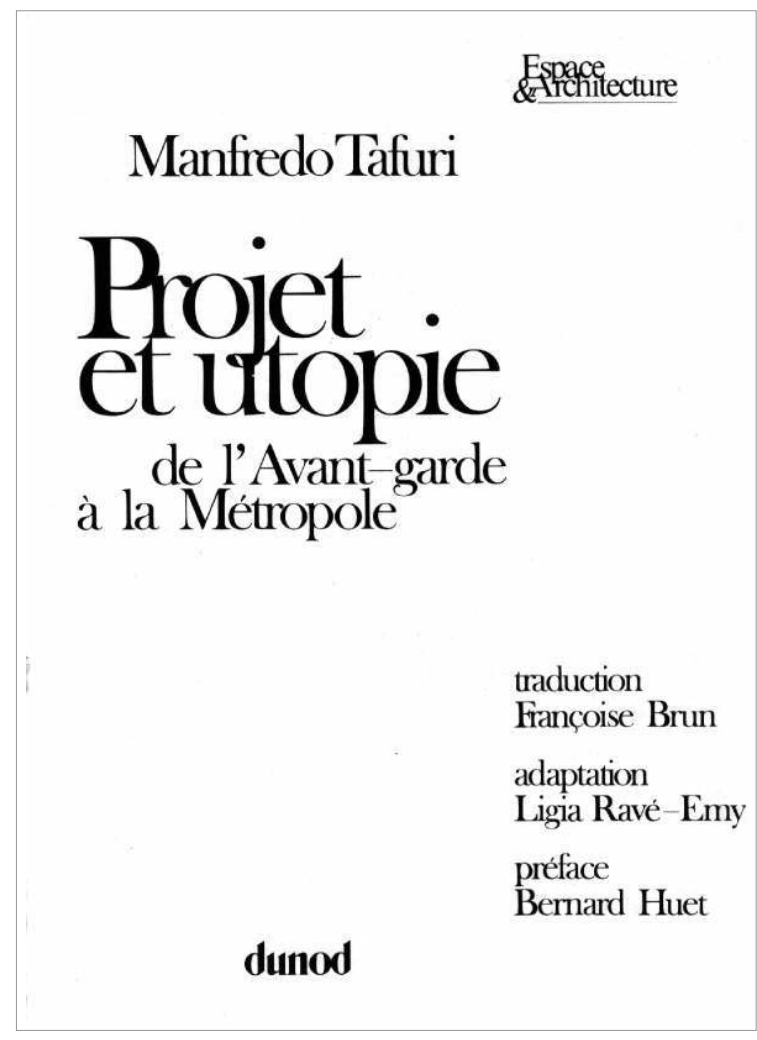

Manfredo Tafuri, Projet et utopie, 1979 [édition originale 1973].
11. Voir notamment les textes les plus caractéristiques des deux premières étapes de l'École de Francfort (Institute für Sozialforschung): Max Horkheimer, «Théorie traditionnelle et théorie critique ", Théorie traditionnelle et théorie critique, Paris, Gallimard, 1974, pp. 15-90; M. Horkheimer et Theodor W. Adorno, La dialectique de la Raison: fragments philosophiques, Paris, Gallimard, 1983.
12. Voir K. Michael Hays, "Critical Architecture: Between Culture and Form », Perspecta, vol. XXI, 1984, pp. 15-29 L'architecture devrait occuper une place entre le produit culturel et la discipline autonome: " a place between the efficient representation of preexisting cultural values and the wholly detached autonomy of an abstract formal system ».
13. Ignasi de Solà-Morales, "Topografía de la arquitectura contemporánea », Diferencias. Topografía de la arquitectura contemporánea, Barcelone, G. Gili, 1995, p. 13. ("Topographies of Contemporary Architecture », in Differences. Topographies of contemporary Architecture, Cambridge, MIT Press, 1997, p. 15.) 
Robert Somol et Sarah Whiting, respectivement professeurs à UCLA et à Harvard, affirmaient de leur côté dans un article paru en 2002 - en se référant il est vrai au contexte américain - que ce projet de criticality, après plusieurs décennies, avait fini par ruiner la discipline elle-même ${ }^{14}$. Et pour eux, le symbole de ce dépérissement avait été l'arrêt de la parution d'Assemblage en 2000, une référence en matière de publication critique, héritière de la revue Oppositions. Comme alternative à cette architecture critique, les deux auteurs proposaient alors une architecture qualifiée de "projective». Si l'architecture critique ambitionnait d'être l'« indicateur » culturel d'une réalité qu'elle matérialisait et critiquait par une forme (et dans certains cas, l'impossibilité de la forme même), l'architecture projective en revanche se fonde sur une pratique professionnelle "plus souple», plus « cool», purement disciplinaire, et pas nécessairement aboutie. C'est la même année que Michael Speaks ${ }^{15}$ proposait de remplacer la " théorie " par une « intelligence créatrice »- design intelligence -, s'appuyant littéralement sur les processus flexibles et adaptables de la nouvelle économie post-capitaliste et, également, sur les méthodes des agences de renseignements pour affronter les menaces globales, c'est-àdire sur une capacité d'adaptation en toute circonstance et en tout lieu. L'alliance de l'intelligence projectuelle, des technologies de conception assistée par ordinateur, de la communication et de l'information, et du marketing, a comme résultat une série de pratiques que l'on pourrait désigner comme " post-critical».

II convient de préciser qu'en cours de route on a oublié que la " théorie critique " s'attaquait déjà à la déshumanisation créée par une certaine raison des Lumières, et que le mythe de l'architecture rationaliste était l'objectif de la critique tafurienne. En ce sens, la marchandisation d'un monde moderne réifié, dont la seule raison était l'instrumentalisation, devait être démantelée non seulement par les disciplines mais aussi et surtout par le sujet critique et réflexif. Ce qui nous incite à penser que nous avons besoin, non pas de moins de critique mais de davantage de critique: soit une reconstruction de la rationalité critique, considérée non comme la recherche d'une adéquation avec ce qui est établi (comme le fait la théorie traditionnelle), mais comme une pensée capable de problématiser ce qui avait été considéré jusqu'ici comme axiomatique; ou encore, pour reprendre les propos d'Alvin Ward Gouldner, comme un mode de "penser notre pensée propre $^{16} "$, comme une réflexion critique sur notre discours et ses fondements. La critique devrait essentiellement s'affirmer, aujourd'hui, comme une métacommunication, un langage. L'architecture, me semble-t-il, devrait être entendue comme une opération intellectuelle (un regard sur le monde) qui va au-delà de la simple résolution acritique de problèmes fonctionnels. L'intellectuel ne peut pas être réduit, ainsi que le rappelait justement Edward W. Said, " au statut de professionnel sans visage, expert compétent dont le seul souci est de faire son travail ${ }^{17} »$. Une place pour la critique est par conséquent possible.

Et si un quelconque mérite devait être accordé à cette attaque contre la théorie, ce serait d'avoir libéré l'architecture critique de l'impossibilité paralysante d'agir positivement, dans un monde dominé par le
14. Robert Somol et Sarah Whiting, " Notes around the Doppler Effect and Other Moods of Modernism », Perspecta n ${ }^{\circ} 33$, The Yale Architectural Journal, 2002, pp. 72-77. On trouve l'histoire de cette crise chez George Baird, " "Criticality" and Its Discontents", Harvard Design Magazine n² 21, 2004/2005, pp. 16-21.

15. Michael Speaks, "Design Intelligence and the New Economy ", Architectural Record, janvier 2002, pp. 72-79; « Design
Intelligence: Or Thinking After the End of Metaphysics », Architectural Design, vol. LXXII, n 5, 2002, pp. 4-6. « Ces pratiques dotées d'une grande intelligence projectuelle sont capables de manipuler le problème posé en vue d'opportunités pouvant être explorées, permettant ainsi un niveau plus élevé d'innovation; de telles pratiques s'adaptent aux conditions changeantes [...], et sont presque toujours moins sensibles aux changements ambiants hostiles » (p. 6). Selon Speaks, « les relations entre le penser et le faire sont chaque fois un peu plus imprécises [...] et cela change le visage de l'architecture ».

16. Alvin Ward Gouldner, The Dialectic of Ideology and Technology, New York, Seabury Press, 1976, p. 49.

17. Edward W. Said, Des intellectuels et du pouvoir, Paris, Seuil, 1996, p. 27. 
Marché. L'expérimentation architecturale liée, entre autres, à l'écologie, au patrimoine, à un nouvel habitat rationnel, à un urbanisme participatif, et grâce à la capacité presque infinie de générer des géométries complexes par ordinateur, a fait exploser les limites trop souvent verrouillées par l'architecture critique. II s'agit de confronter cette expérimentation à une architecture soumise aux lois du Marché, qui avait confiné la critique dans un recoin, aux côtés de la théorie. Par conséquent, une " architecture réflexive » est possible, se situant dans la rue, faisant face aux problèmes réels, qui n'oublie pas la pensée critique et qui est capable de s'opposer aux architectures abstraites de laboratoire ${ }^{18}$.

\section{Pour la critique de l'architecture}

La théorie pose les bases du discours, et à partir de ces prémisses, la critique essaie de montrer de quelle manière les problèmes sont mis en rapport avec leurs solutions ${ }^{19}$. L'une est abstraite, I'autre analyse au cas par cas. Rappelons que le terme de « critique » vient du grec krinein : distinguer, différencier ; critiquer, c'est faire des distinctions, car tout n'est pas valable également. Cette critique émerge lorsque nous devons formuler et justifier les critères qui ont été retenus pour construire un jugement. Certains critères sont évidents : I'architecture devrait satisfaire le programme du client et protéger des intempéries. Mais il y en a bien d'autres, issus du monde complexe qu'est l'architecture. William S. Saunders, directeur de la revue Harvard

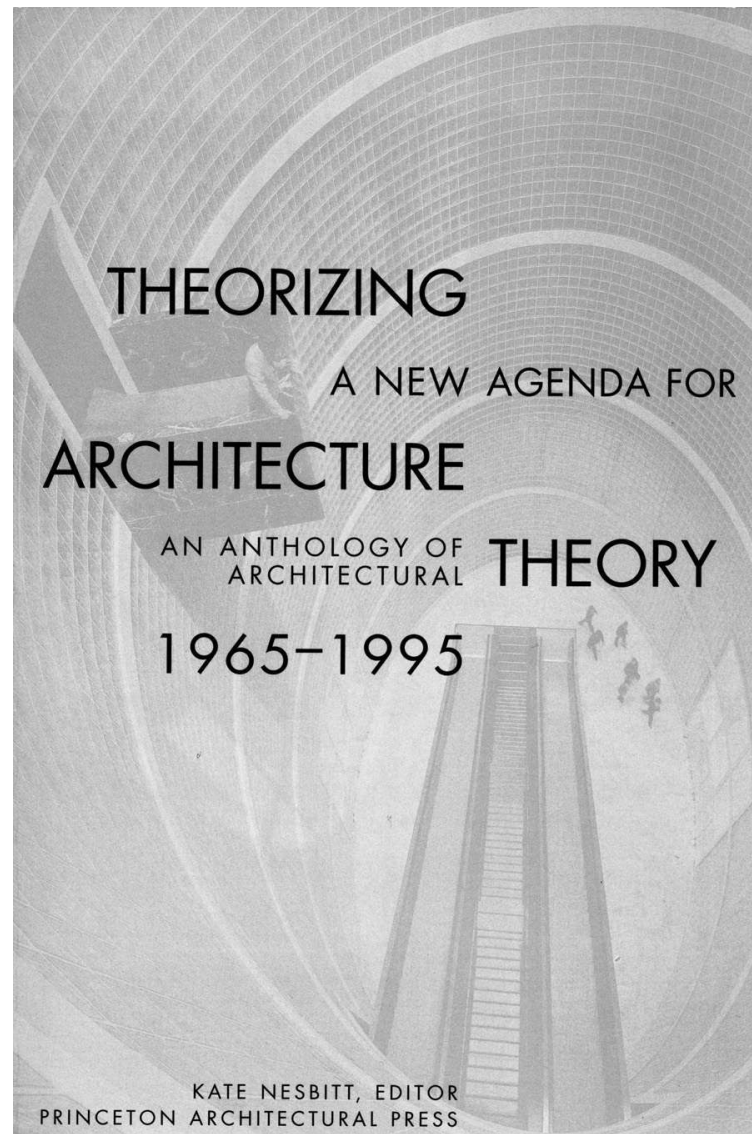

Kate Nesbitt,

Theorizing a New Agenda for Architecture, 1996.

Design Magazine, en a proposé un certain nombre: "L'environnement construit devrait: se rapprocher de l'art; créer de la beauté; réussir des expériences

18. C'est la proposition, notamment, d'Arie Graafland dans son texte "On Criticality », in A. Graafland et Leslie Jay Kavanaugh (dir.), Crossover. Architecture Urbanism Technology, Rotterdam, 010 Publishers, 2006, p. 702-703.

19. Pierre Bourdieu écrit: «L'histoire de la critique [...] n'a pas d'autre fin que de tenter de porter à la conscience de celui qui l'écrit et de ses lecteurs les principes de vision et de division qui sont au principe des problèmes qu'ils se posent et des solutions qu'ils leur apportent » (Les Règles de l'art. Genèse et structure du champ littéraire, Paris, Seuil, coll. "Points essais », 1992, p. 272). Bien qu'en référence à la littérature, il est facile d'appliquer cette réflexion à l'art en général. 


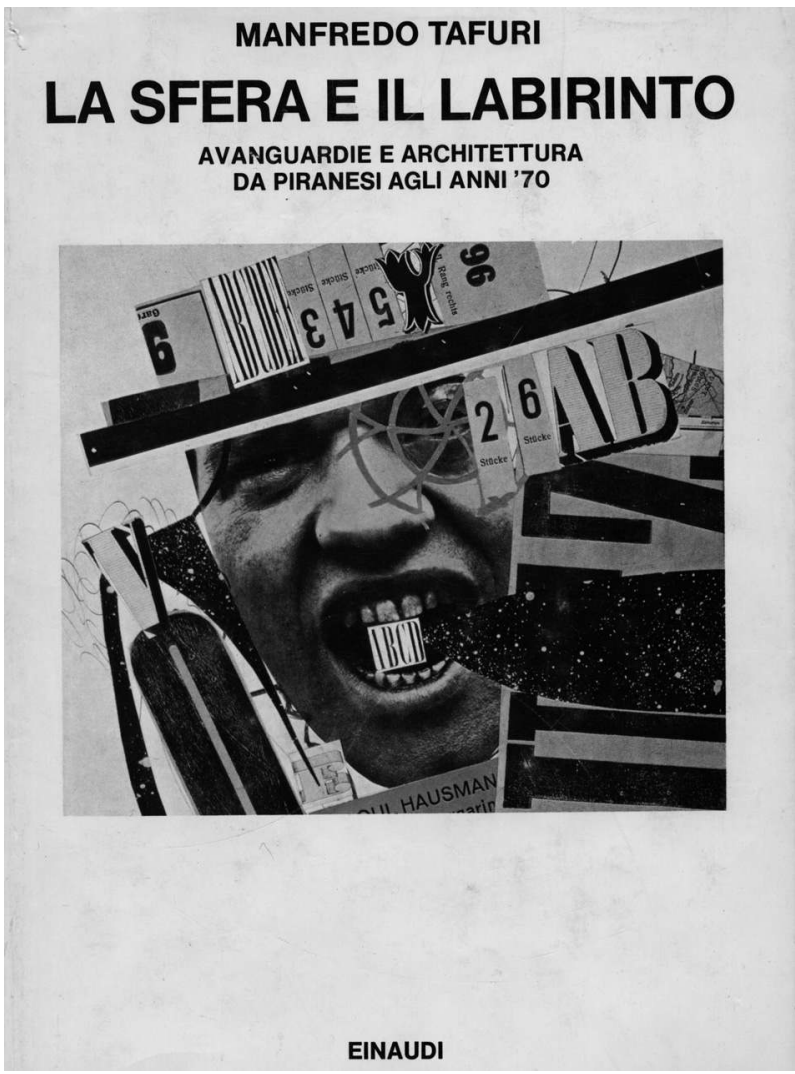

Manfredo Tafuri,

La Sfera e il Labirinto,

1980.

Peter Blundell Jones, Doina

Petrescu

et Jeremy Till, Architecture and

Participation,

2005.

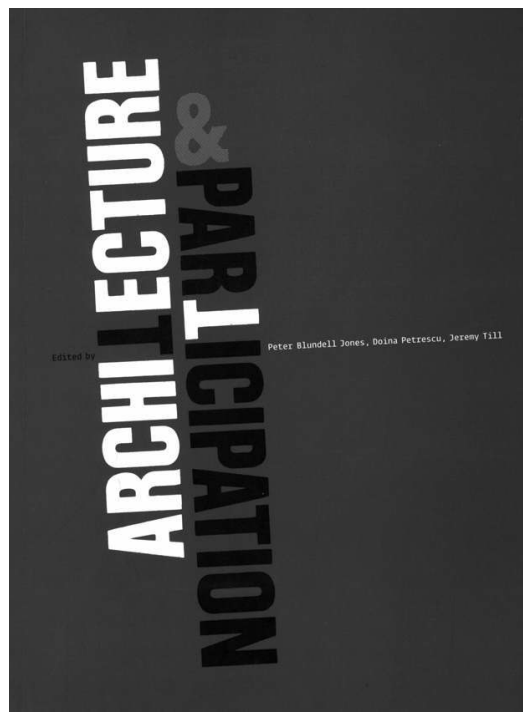

Perspecta $\mathrm{n}^{\circ} \mathbf{3 8}$ 2006. 
visuelles satisfaisantes au moyen de l'échelle, de la proportion, de l'équilibre, du rythme, de la texture, de la couleur, de la variation, du modèle; harmoniser la fonction avec l'image et le symbolisme; être original ; s'intégrer discrètement dans le milieu environnant; répondre aux caractéristiques de sa région et de son climat; servir de soutien et d'exemple pour les objectifs sociopolitiques et les conduites morales; exprimer les idéaux de sa communauté/société; ne pas causer de dommages à l'écosystème ; être bien construit; satisfaire les aspirations du client (et pas l'idée que se fait I'architecte de ce que son client devrait vouloir); mener à bien les missions de l'architecte; être durable; apporter du confort physique et psychique; atteindre économiquement ses buts; réussir le retour sur investissement; inciter les gens à le visiter et à y revenir ; contribuer à une haute productivité ${ }^{20} \ldots$ » Et il faut encore y ajouter les contradictions et les conflits inéluctables entre une architecture plus « proche » de l'art, ou de la «profession», ou plus « intellectuelle», ou considérée comme un "service".

Le fait que l'architecture soit le résultat d'une synthèse de critères fort nombreux et divers rend nécessaire sa fragmentation en ses différentes composantes, afin de procéder à des analyses séparées. Ces composantes, Tafuri les répertoriait comme des « rapports sur mesure, des horizons symboliques, des hypothèses d'avant-garde, des structures de langage, des méthodes de restructuration de la production, des inventions technologiques ${ }^{21}$ ». Ensuite, après avoir été historicisés, ces éléments se recomposent en une "construction historique »-c'est en cela que consiste la critique - qui rend compte de la totalité de l'œuvre. Et dans tous les cas de figure, I'histoire critique est toujours « un projet de démythification $^{22}$ ".

Ce processus donne lieu à une construction critique provisoire, dans la mesure où il s'agit d'une tâche finalement incertaine. Citons à nouveau Tafuri : «Une histoire vraie n'est pas celle qui s'appuie sur des preuves philologiques indiscutables, mais celle qui recueille son propre arbitraire, qui se reconnaît comme édifice incertain. » La critique résultante sera contingente - loin alors des formes systématiques de l'Histoire - et tendra vers un véritable projet historique ${ }^{23}$ : un projet, parce qu'en construisant une nouvelle synthèse, on « inventera » un autre objet par son interprétation, et comme ce projet sera toujours provisoire, ses hypothèses seront révisables et ses réussites dépassables.

Loin d'être de l'ordre du trivial, la description de ce qui est visible correspond à une question théorique importante: il est en effet nécessaire de disposer d'une pensée architecturale pour comprendre où se situe l'intérêt de toute œuvre, au-delà de sa raison historique. Et il est nécessaire, en conséquence, de disposer de " critères de pertinence " pour se mesurer à l'analyse critique. C'est le moment d'une certaine prise de position: structuralisme, pragmatisme, phénoménologie, déconstruction, psychologisme, formalisme... correspondent à des points de vue non exclusifs, parfois complémentaires; ils constituent des observatoires à partir desquels il est possible d'observer, intentionnellement, le fait architectural. En cherchant à atteindre une certaine objectivité et un jugement équilibré, il s'agit $d^{\prime}$ 'analyser et de reconstruire la raison compositive et
20. William S. Saunders, «From Taste to Judgment », Harvard Design Magazine, $\mathrm{n}^{\circ} 7$, 1999 , p. 2. Voir également W. S. Saunders, The New Architectural Pragmatism. A Harvard Design Magazine Reader, Minneapolis, University of Minnesota Press, 2007.

21. Manfredo Tafuri, « Il progetto storico », introduction à La Sfera e il Labirinto, Turin, Einaudi, 1980, p. 20. Finalement, «la spécificité de l'architecture réside dans sa façon d'établir une relation entre les différentes structures qui convergent vers elle »: M. Tafuri, Théories et histoire de l'architecture, Paris, SADG, 1976, p. 281.

22. «[...] le terme de reconstruction impliquerait les questions de "vrai ou faux" que je ne considère pas comme opportunes. Il est évident que "reconstruction" implique une réalité préexistante qui doit être façonnée, et je ne suis pas d'accord là-dessus; il vaut mieux parler de construction historique »: Mercedes Daguerre et Giulio Lupo, «Entrevista con Manfredo Tafuri », Materiales, n 5, Buenos Aires, PEHCH-CESCA, mars 1985, p. 25. Sur la critique démythificatrice, voir Demetri Porphyrios, "On Critical History », in Joan Ockman (dir.), Architecture, Criticism, Ideology, Princeton (NJ), Princeton Architectural Press, 1985, pp. 16-22.

23. M. Tafuri, « Il progetto storico », op. cit. note 21, p. 17. 
constructive de l'acte architectural, en contribuant à en dévoiler la logique. Pour trouver cette logique de composition, présente dans chaque œuvre, sans doute faudrat-il I'aide des archives, des théories dominantes, des poétiques personnelles des auteurs, des relations avec les clients, de la connaissance des raisons culturelles, économiques et politiques du moment... II sera également indispensable de connaître les péripéties du projet du processus constructif et les modifications subies jusqu'à la fin du processus. À l'approche historique "objective ", il conviendra d'ajouter l'interprétation de toutes ces sources et le parcours des intentionnalités, avec ses succès et ses échecs. Et enfin, il sera nécessaire de connaître et d'expérimenter, de préférence directement, l'objet architectural.

Rappelons, particulièrement après Tafuri, que la critique ou l'histoire ne peuvent préfigurer des solutions nouvelles, parce qu'elles ne peuvent pas franchir ce pas. Elles représentent néanmoins, et c'est déjà important, " une contestation permanente du présent ${ }^{24}$ ». Leur mission doit être la démythification des œuvres architecturales passées et présentes, en soulignant leurs contradictions. Pour y parvenir, les processus de leur fabrication, de leur production et de leur utilisation devront être analysés grâce à une dissection méthodique qui, au final, aura fait exploser la synthèse de l'œuvre ${ }^{25}$.

\section{Vers une théorie de l'architecture}

Critique et théorie sont une lorsque, comme le souligne Terry Eagleton, « c'est cette réflexion critique que nous connaissons comme théorie. Ce type de théorie advient lorsque nous sommes dans l'obligation de prendre conscience, de façon inhabituelle, de ce que nous sommes en train de faire ${ }^{26}$. " Mais il n'existe pas " une seule » théorie, tout comme il n'existe pas " une seule » architecture. II n'y a pas, par conséquent, un système fermé, unique, mais des phénomènes de discontinuité, de superposition et de juxtaposition qui substituent aux anciens mécanismes dominants de l'Académie des plates-formes provisoires et fragiles ${ }^{27}$.

Reste que la théorie ne peut pas se situer en marge de la pensée et des débats contemporains; elle devrait en revanche s'éloigner de toute tentation d'instrumentalisation. De fait, rien dans l'étymologie du mot " théorie » ne nous indique qu'elle devrait avoir une corrélation immédiate avec la pratique. II est vrai que certains soutiens relativement récents dans les champs de la philosophie ou de la critique littéraire (comme la déconstruction derridienne ou la conceptualisation deleuzienne) ont converti la théorie architecturale en une alliée directe de pratiques prétendument avantgardistes. Et il semblerait que la théorie - oubliant alors la dénonciation que Tafuri faisait de la " critique opératoire " - ait ainsi reproduit les dérives instrumentales de la Modernité : lorsque par exemple réflexion coïncidait avec opération, lorsque la théorie n'était rien de plus que de l'idéologie et qu'elle remplaçait « la rigueur analytique par des jugements de valeur déjà constitués, utilisables pour l'action immédiate ${ }^{28}$ ». Ce serait oublier - pour continuer avec Tafuri - que la critique est aussi Histoire, et qu'en tout cas elle pose des questions mais n'avance pas de solutions, dans un processus dans lequel l'architecture se manifeste toujours dans une transformation permanente.
24. Voir M. Tafuri, « Les tâches de la critique », chap. VI, Théories et histoire [...], op. cit. note 21

25. Voir M. Tafuri, « Il progetto storico», op. cit. note 21, p. 20.
26. Terry Eagleton, After Theory, Londres, Allen Lane, 2003, p. 27

27. Voir I. de Solà-Morales, "Arquitectura débil » et «Sadomasoquismo. Crítica y práctica arquitectónicas », Diferencias, op. cit. note 13 ("Weak Architecture » et « Sadomasochism: Criticism and Architectural Practice », Differences. Topographies of contemporary

Architecture, Cambridge, MIT Press, 1997).
28. M. Tafuri, Théories et histoire [...], op. cit. note 21, p. 209. 
William S. Saunders,

The New

Architectural

Pragmatism, 2007.

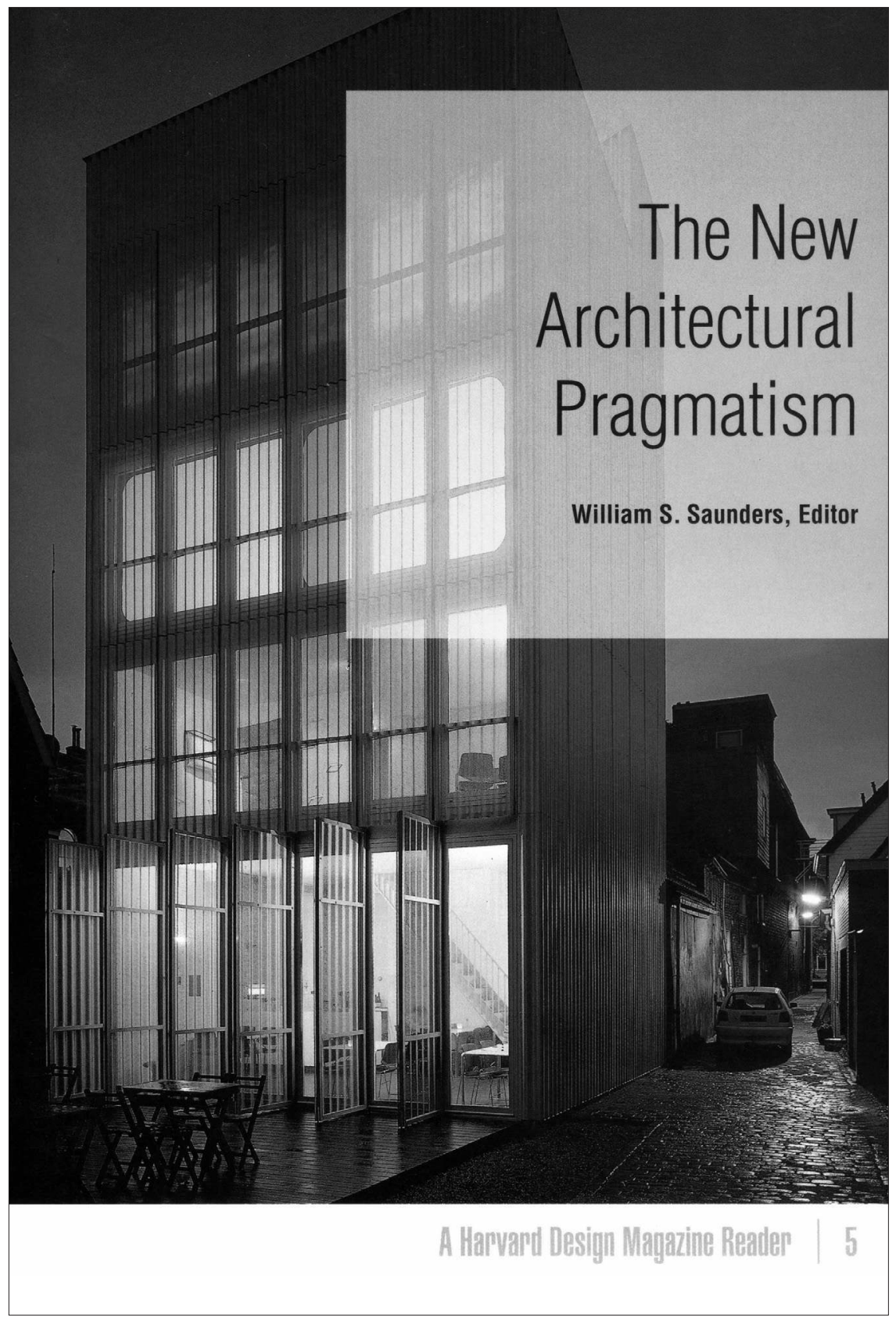




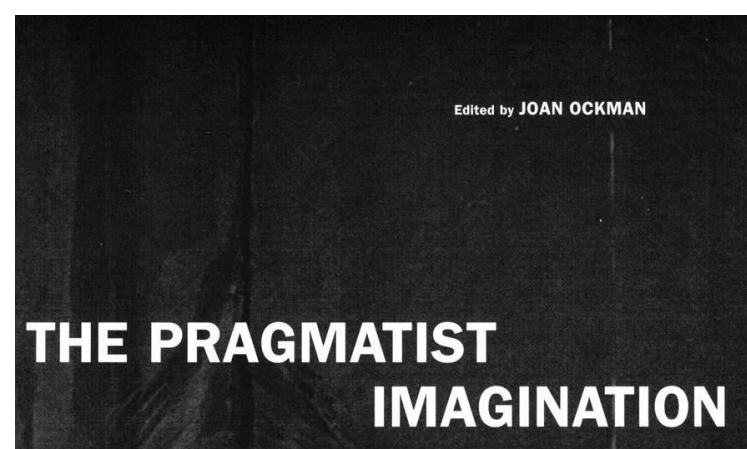

THINKING ABOUT "THINGS IN THE MAKING"

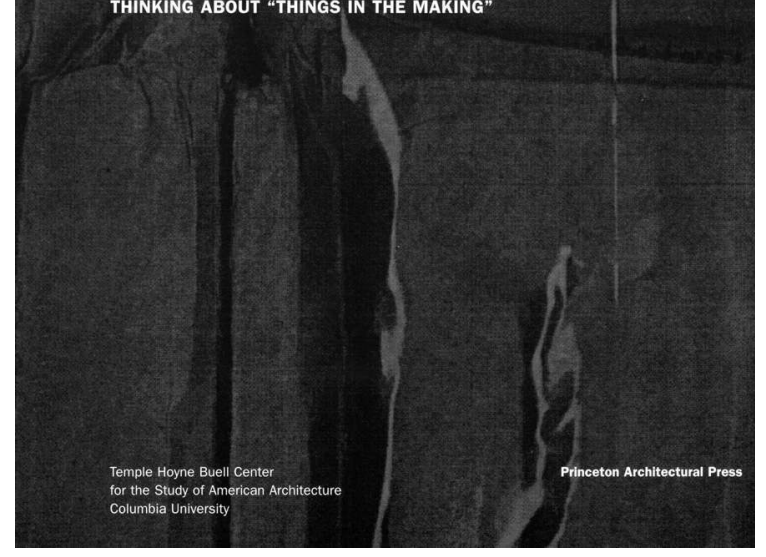

Joan Ockman,

The Pragmatist

Imagination, 2000.
Le rôle de la critique dans l'architecture contemporaine s'affirmera, par exemple, non pas pour apporter une réponse formelle et concrète à un problème architectural, mais pour bousculer l'architecture elle-même, pour l'interroger, pour examiner ses faiblesses et ses défauts structurels. Dans ses textes sur la déconstruction, Mark Wigley a insisté sur cet aspect: «Traduire la déconstruction dans l'architecture ne conduit pas simplement à une reconfiguration formelle de l'objet; il s'agit plutôt de mettre en question la condition de l'objet, son objectalité, de problématiser la condition de l'objet sans l'abandonner ${ }^{29}$."

Il est très étonnant de noter que le regain de la théorie dans les années 1970 a été justement provoqué par une génération qui souhaitait récupérer, au profit de la profession, la dimension intellectuelle perdue au cours des années précédentes. Mais le résultat de cette récupération - via les thèses du post-structuralisme français - a été que la théorie de l'architecture tombe dans certains excès, dans des hermétismes et des obscurantismes conduisant à une sorte d' " industrie culturelle » quasiment autonome, " avec son aura, ses étoiles et ses modes », et qui doit déjà être déconstruite ${ }^{30}$. De quelle manière? En retournant à l'architecture elle-même.

Citons à nouveau Wigley, lors de l'un des débats menés à la conférence «The Politics of Contemporary Architectural Discurse » (Tulane University, La NouvelleOrléans, 1995), et où il affirmait ceci : « Je crois que l'architecture est incroyablement intéressante. Les tentatives désespérées pour instrumentaliser la théorie reposent toujours sur la prémisse selon laquelle l'architecture est stupide, qu'elle requiert de se revêtir de théo-
29. Mark Wigley, «The Translation of Architecture. The Production of Babel», Assemblage, n॰8, 1989, p. 18. Dans le catalogue de l'exposition Deconstructivist Architecture organisée au MoMA de New York en 1988, Wigley affirmait: «Un architecte déconstructiviste n'est donc pas celui qui démolit des bâtiments, mais celui qui localise les dilemmes inhérents aux édifices », in Ph. Johnson et M. Wigley, Deconstructivist Architecture, New York, Museum of Modern Art, 1988, p. 11. Avec la déconstruction, il ne s'agit pas, par conséquent, d'établir des stratégies ou des jeux formels (ce n'est pas une méthode de production), mais de modifier, de trouver ce qui est caché, de libérer ce qui est surprenant.

30. Voir le texte de Joan Ockman, "Untitled », Assemblage, n 41, avril 2000, p. 61.

31. M. Wigley, Assemblage, $n^{\circ} 27$, août 1995, p. 101. Voir dans le même numéro les actes de la conférence, sous le titre "Tulane Papers: The Politics of Contemporary Architectural Discourse ».

32. Comme l'écrit Antonio Monestiroli: «[...] un projet sans théorie est privé de sa raison constitutive et peut donc se modifier de multiples façons sans conséquences sérieuses [...]. Si l'on veut revendiquer un rôle actif dans la construction de la réalité que le projet $d^{\prime}$ architecture mène à bien, il est nécessaire de mettre en valeur ses 
ries extraordinaires empruntées à la science, à la philosophie, à la mécanique des automobiles, ou à quoi que ce soit d'autre. Je crois que l'image traditionnelle de I'architecture est beaucoup plus intéressante ${ }^{31}$. »

Être d'accord avec le rejet d'une théorie instrumentalisée qui justifie et masque à l'occasion de mauvaises architectures est une chose, mais est-ce que cela signifie pour autant que la théorie ne devrait pas exister? Devons-nous nous résigner au fait que le projet architectural n'a plus besoin de se justifier désormais ${ }^{32}$ ? Car, en définitive, la théorie est la seule voie qui permet sa transmission: un langage partagé (qui reste, naturellement, très éloigné $d^{\prime} u n$ code obligatoire).

Nous avons vu, au début de cet article, que la critique la plus féroce de la théorie était venue du néo-pragmatisme. Or c'est précisément dans le « premier pragmatisme » (Charles Peirce, William James, John Dewey) que I'on peut trouver quelques-uns des éléments permettant de combattre le plus clairement le discrédit de la théorie. C'est en tout cas l'intention qui avait été affichée par les organisateurs du colloque de la Columbia University de New York en avril $2000^{33}$ : autour de la défense d'une certaine "imagination pragmatique ", les participants avaient ainsi tenté de démonter le principe de l'opposition entre une architecture américaine (pragmatique et sans idées) et une architecture européenne (plus théorique). Parmi les thèses du premier pragmatisme analysées lors du colloque, l'une des plus représentatives était sans doute celle défendue par William James: "Ce qui existe réellement, ce ne sont pas les choses faites mais les choses en train de se faire [...]. La philosophie devrait explorer cette compréhension vivante du mouvement de la réalité ${ }^{34}$. M Mais l'un des problèmes posés par la pensée pragmatique, c'est celui de ne pas considérer qu'à travers la connaissance il y a toujours le désir de domination. Dans sa critique du rationalisme positiviste, Jürgen Habermas cherche à échapper à cette situation en proposant une théorie de l'agir communicationnel, dans laquelle le monde objectif doit être complémentaire du monde social et du monde subjectif. Ainsi l'interaction entre les individus permettrait-elle de relativiser " mon » interprétation, en la confrontant à celle des " autres » pour pouvoir arriver à des consensus ${ }^{35}$.

Pour l'architecture et I'urbanisme, cela aboutit à placer au premier plan les questions de la temporalité, de la modification et des stratégies ouvertes du projet, de la réhabilitation de la politique (de la res publica) et de la participation collective, face aux esthétiques imposées. Ainsi, nous pourrions avoir des modes de construction intemporels, des programmes ouverts, des procédures flexibles et une culture de redistribution plutôt que de développement ${ }^{36}: c^{\prime}$ est-à-dire des stratégies capables de re-politiser la société et de démocratiser l'architecture. Et pour ce qui concerne la participation citoyenne dans le projet, une fois dépassées les sempiternelles critiques élitistes de la part des " experts », il s'agirait de chercher à établir une négociation autour de l'espace, au-delà des processus autoritaires et dominants du projet ${ }^{37}$. Cette architecture faite pour les gens et par les gens, socialement engagée, retrouverait une certaine qualité éthique face à l'imposition purement visuelle de ces «monuments mercantilistes » qui, dans leur quête d'un impact instantané, sont à la fois franchement non durables et rapidement raisons essentielles [...]. Autrement, tout apparaît comme licite »: "Necessità della teoria », in Paolo Portoghesi et Rolando Scarano (dir.), Il progetto di architettura. Idee, scuole, tendenze all'alba del nuovo millennio, Rome, Newton \& Compton editori, 1999, p. 381.

33. J. Ockman, The Pragmatist Imagination: Thinking about Things in the Making, New York, Columbia University/Princeton Architectural Press, 2000.
34. William James, "Bergson and His Critique of Intellectualism », 1909, cit. ibid., p. 27.

35. Voir Jürgen Habermas, Teoría de la acción comunicativa I, Madrid, Taurus, 1987, pp. 136 146. Théorie de l'agir communicationnel. Tome I: Rationalité de l'agir et rationalisation de la société. Paris, Fayard, 1981/1987. [Titre original: Theorie des kommunikativen Handelns, 2 tomes, 1981.]
36. Voir Hashim Sarkis, "On the Line between Procedures and Aesthetics », in J. Ockman, op. cit. note 33, pp. 92-103.

37. Voir, dans I'abondante bibliographie sur ce point, Peter Blundell Jones, Doina Petrescu et Jeremy Till, Architecture and Participation, Londres/New York, Spon Press, 2005. Et sur les principes de l'« espace public », voir la Charte de Principes du Forum Social Mondial, 2001, sur le site: www.forumsocialmundial.org.br. 
superflus. C'est une architecture qui, selon Juhani Pallasmaa, cherche à être aussi une " architecture pour les sens 38 ", proche des propositions "tranquilles», transposées aux lieux, de Glenn Murcutt.

II me semble que l'un des problèmes posés par l'architecture contemporaine, c'est le peu de place qu'elle laisse à la théorie. Ashley Schafer a proposé récemment une "théorie après l'après-théorie ", susceptible de libérer précisément la théorie de son instrumentalisation par la pratique, et de la transformer en une plate-forme permettant $d$ ' « informer la manière dont nous travaillons plutôt que de dicter ce que nous faisons ${ }^{39}$ ". Une théorie qui peut être « développée, changée et modifiée dans le temps ", loin de constituer un manifeste. II s'agit par conséquent d'une idée de théorie qui n'est pas prescriptive (comme cela l'avait été d'Alberti à Peter Eisenman) mais critique par rapport à la situation de l'architecture actuelle, proche de l'« histoire critique » de Tafuri. Une théorie contingente et ouverte pourrait être la réponse au refus ou à la négation de la théorie.

L'architecture, libérée des discours parallèles, d'un verbiage ronflant et incompréhensible, et comprise davantage comme une activité capable de négocier avec le temps, devient le fruit de la mise en relation des formes projetées et construites et des idées qui les ont nourries. Ces idées et ces valeurs culturelles sont nombreuses, ce qui ne signifie pas que nous devions toutes les valider. Cette multiplicité constitue seulement le point de départ de futures légitimations, et non pas une légitimité acritique définitive ${ }^{40}$. À partir du moment où, par chance, l'Académie n'existe plus, pas plus que le projet absolu, ou le système unique de règles, il n'est pas possible d'identifier des principes premiers ou des fondements inamovibles. Toutefois, la cohérence doit exister: même le chaos a ses règles! Si l'architecture est une façon de penser le monde, de penser notre temps, cette pensée devrait pouvoir se transmettre ; et dans ce processus, les discours occultés ou non explicites devraient également refaire surface. La raison et l'intuition, ce qui relève de l'objectif et du subjectif, les questions de forme et de contenu, les expériences et les attentes, la connaissance tacite et articulée... : les deux termes de chaque paire devraient être explicités, racontés, soumis à la réflexion, critiqués, partagés voire rejetés. C'est ce que l'on appelle (une) Théorie de l'Architecture.

À la mémoire de Rafael González Sandino*

TRADUCTION DE L'ESPAGNOL PAR LILIAN PÉRIER
38. Juhani Pallasmaa, The eyes of the skin. Architecture and the senses (préface de Steven Holl), Chichester, John Wiley \& Sons, 2005. Voir également l'article de J. Pallasmaa, "Toucher le monde », dans le $n^{\circ}$ 20/21 des Cahiers de la recherche architecturale et urbaine, pp. 223-233.

39. Ashley Schafer, «Theory After (After-Theory) », Perspecta, $n^{\circ}$ 38, "Architecture After All », 2006, p. 110.
40. Voir l'introduction de Marco Diani et Catherine Ingraham, Restructuring Architectural Theory, Evanston, Illinois, Northwestern University Press, 1989, p. 1 sq.
* Rafael González Sandino est mort le 4 mai 2008. En tant que professeur titulaire de théorie de l'architecture du département Histoire, Théorie et Composition architecturales de l'école d'architecture de l'université de Séville, il a formé toute une génération d'architectes. Ses thèmes de réflexion ont porté en particulier sur les rapports entre la pensée philosophique et l'architecture, sur le patrimoine et l'environnement. 